\title{
ON THE COMPLETENESS OF A CERTAIN METRIC SPACE WITH AN APPLICATION TO BLASCHKE'S SELECTION THEOREM ${ }^{1}$
}

\section{G. BALEY PRICE}

1. Introduction. The purpose of this note is to prove that the metric space whose elements are the closed, bounded, non-null subsets of a complete metric space, and whose metric is the Hausdorff distance, is complete; and, using this result and others already known, to give a simple proof of Blaschke's selection theorem.

2. Preliminaries. Let $K$ be a metric space with elements $x, y, \ldots$ and distance function $d(x, y)$. A sequence $x_{1}, x_{2}, \cdots$ in $K$ such that $\sum_{1}^{\infty} d\left(x_{i}, x_{i+1}\right)$ converges has been called an absolutely convergent sequence by MacNeille ${ }^{2}$ [7, p. 192]. Every absolutely convergent sequence is a Cauchy sequence, and every Cauchy sequence contains absolutely convergent subsequences.

Let $K^{*}$ be a metric space whose elements $X, Y, \cdots$ are the closed, bounded, and non-null subsets of $K$, and whose distance function $D(X, Y)$ is the Hausdorff distance between the sets $X$ and $Y$ (see Hausdorff [5, pp. 145-146] and Kuratowski [6, pp. 89-90]).

3. The theorem. If $K$ is complete, then $K^{*}$ is also complete.

Let $X_{1}, X_{2}, \cdots$ be any Cauchy sequence in $K^{*}$; without loss of generality we can assume that it is absolutely convergent. We shall define a set $X$ and show that it is the limit of the given sequence. Let $x_{1}$ be any point in $X_{1}, x_{2}$ any point in $X_{2}$ such that $d\left(x_{1}, x_{2}\right)<D\left(X_{1}, X_{2}\right)$ $+2^{-1}, x_{3}$ any point in $X_{3}$ such that $d\left(x_{2}, x_{3}\right)<D\left(X_{2}, X_{3}\right)+2^{-2}$, and so on. The existence of points $x_{2}, x_{3}, \cdots$ with the properties stated follows from the definition of the Hausdorff distance. Every point $x_{i}$ in $X_{i}$ is a member of a sequence $x_{1}, x_{2}, \ldots$ of the kind described. The sequence $x_{1}, x_{2}, \ldots$ is absolutely convergent and hence a Cauchy sequence; since $K$ is complete, it has a limit $x_{0}$ in $K$. Let $X_{0}$ be the locus of all the points $x_{0}$ obtained as the limits of all possible sequences formed in the manner stated; let $X$ be the closure of $X_{0}$. Then $X$ is closed, bounded, and non-null, and $X$ is in $K^{*}$. We shall show that $\lim X_{k}=X$. Let any $\epsilon>0$ be given. Choose $n=n(\epsilon)$ so that $\sum_{n}^{\infty}\left[D\left(X_{i}, X_{i+1}\right)+2^{-i}\right]<\epsilon / 2$. Let $x^{*} \varepsilon X$, and let $x_{0}$ be the limit of a

${ }^{1}$ Presented to the Society, December 28, 1938, under the title Spaces whose elements are sets.

${ }^{2}$ Numbers in square brackets refer to the references at the end. 
sequence $x_{1}, x_{2}, \cdots, x_{k}, \cdots$ and such that $d\left(x^{*}, x_{0}\right)<\epsilon / 2$. Then the distance from $x^{*}$ to $X_{k}$ is equal to or less than

$$
\begin{aligned}
d\left(x^{*}, x_{0}\right)+\sum_{k}^{\infty} d\left(x_{i}, x_{i+1}\right) & <d\left(x^{*}, x_{0}\right)+\sum_{k}^{\infty}\left[D\left(X_{i}, X_{i+1}\right)+2^{-i}\right] \\
& <\epsilon / 2+\epsilon / 2=\epsilon
\end{aligned}
$$

if $k \geqq n$. Since every point $x_{k}$ in $X_{k}$ belongs to a sequence $x_{1}, x_{2}, \cdots$, the distance from any point $x_{k}$ to $X$, which does not exceed the distance from $x_{k}$ to the limit $x_{0}$ of the sequence $x_{1}, x_{2}, \cdots, x_{k}, \cdots$, is equal to or less than

$$
\sum_{k}^{\infty} d\left(x_{i}, x_{i+1}\right)<\sum_{k}^{\infty}\left[D\left(X_{i}, X_{i+1}\right)+2^{-i}\right]<\epsilon / 2
$$

if $k \geqq n$. From these facts it follows that $D\left(X_{k}, X\right)<\epsilon$ for $k \geqq n$, and hence that $\lim X_{k}=X$. Thus the (absolutely convergent) Cauchy sequence $X_{1}, X_{2}, \cdots$ in $K^{*}$ has the limit $X$ in $K^{*}$, and the proof of the theorem is complete.

4. The space $K^{*}$ when $K$ is a Banach space. The space $K^{*}$ has additional properties when $K$ is a Banach space, that is, a space which is linear, normed, and complete (see Banach $[1$, p. 53]). Let $a X$ denote the set of elements $a x, x \varepsilon X$, when $a$ is a real number; let $X+Y$ denote the set of elements $x+y, x \varepsilon X$ and $y \varepsilon Y$; let $C[X]$ denote the closed convex extension of $X$; and let $\rho(X)$ denote the diameter of $X$. Then $K^{*}$ has, in addition to its elementary properties as a metric space, the following ones:

$$
\begin{aligned}
D(a X, a Y) & =|a| D(X, Y) \quad \text { for every real number } a \\
D\left(X_{1}+\cdots+X_{n}, Y_{1}+\cdots+Y_{n}\right) & \\
& \leqq D\left(X_{1}, Y_{1}\right)+\cdots+D\left(X_{n}, Y_{n}\right) \\
D(C[X], C[Y]) & \leqq D(X, Y) \\
D\left(X+Y_{1}, X+Y_{2}\right) & \leqq D\left(Y_{1}, Y_{2}\right) ; \\
\rho\left(X_{i}\right) & \leqq \rho\left(X_{1}+X_{2}\right) \leqq \rho\left(X_{1}\right)+\rho\left(X_{2}\right), i=1,2 ; \\
\rho(C[X]) & =\rho(X), \quad D(C[X], 0)=D(X, 0) .
\end{aligned}
$$

The last two of these relations have been given by Birkhoff [ 2 , pp. $368,360]$. The proofs of the others will be given elsewhere. It can be shown by means of examples that the inequality may hold in (4.4).

If the limit of a sequence $X_{1}, X_{2}, \cdots$ of convex sets in $K^{*}$ is a set $X$, it follows from (4.3) that $X$ also is convex. For 
$D\left(X_{i}, C[X]\right)=D\left(C\left[X_{i}\right], C[X]\right) \leqq D\left(X_{i}, X\right)$; and since $D\left(X_{i}, X\right) \rightarrow 0$, $\lim X_{i}=C[X]$. But since a sequence has a unique limit, we have $C[X]=X$, and $X$ is convex.

5. Blaschke's selection theorem. Let $E$ be a closed and compact subset of a Banach space $K$, and let $E^{*}$ denote the subset of $K^{*}$ which consists of the closed, non-null subsets of $E$. Then both $E$ and $E^{*}$ are totally bounded, and $E^{*}$ is closed and compact in $K^{*}$ (see Hausdorff [5, pp. 107-108] and Kuratowski [6, p. 91]). Let $E_{C}^{*}$ denote the subset of $E^{*}$ which consists of convex sets. Since $E^{*}$ is totally bounded, any infinite set of elements in $E^{*} \subset E^{*}$ contains a Cauchy sequence; since $K^{*}$ is complete and $E^{*}$ is closed, this sequence has a limit in $E^{*}$. By the result at the end of the last section, this limit element is itself a closed, convex set and therefore belongs to $E_{C}^{*}$. We have thus shown that $E^{*}$ is closed and compact. This result is Blaschke's selection theorem extended to a Banach space (see Blaschke [3] and Bonnesen and Fenchel $[4$, p. 34]).

\section{REFERENCES}

1. S. Banach, Théorie des Opérations Linéaires, Monografje Matematyczne, vol. 1, Warsaw, 1932.

2. G. Birkhoff, Integration of functions with values in a Banach space, Transactions of this Society, vol. 38 (1935), pp. 357-378.

3. W. Blaschke, Kreis und Kugel, Leipzig, Veit, 1916.

4. T. Bonnesen and W. Fenchel, Theorie der konvexen Körper, Ergebnisse der Mathematik und ihrer Grenzgebiete, vol. 3, no. 1, 1934.

5. F. Hausdorff, Mengenlehre, Berlin and Leipzig, de Gruyter, 2d edition, 1927.

6. C. Kuratowski, Topologie I, Monografje Matematyczne, vol. 3, Warsaw, 1933.

7. H. M. MacNeille, Extensions of measure, Proceedings of the National Academy of Sciences, vol. 24 (1938), pp. 188-193.

UNIVERSITY OF KANSAS 